\title{
Giant Basilar Artery Aneurysms Encorporating the Posterior Cerebral Artery: Bypass Surgery and Coil Occlusion
}

\author{
-Two Case Reports-
}

\author{
Christian EWALD, Dieter KÜHNE*, and Werner HASSLER \\ Department of Neurosurgery, Städtische Kliniken, Duisburg, Germany; \\ ${ }^{*}$ Department of Neuroradiolosy, Alfried-Krupp-Krankenhaus, Essen, Germany
}

\begin{abstract}
Giant aneurysms of the basilar artery are rare. With a diameter of $25 \mathrm{~mm}$ or more they are often partially thrombosed and show atheromatous plaques. There are some problems in the treatment especially when the aneurysm is broadbased with bulbous origin encorporating the origin of the posterior cerebral artery (PCA). In many of these cases neither operative clipping alone nor coil embolization alone will be practical without causing an ischemia in the depending brain areas. We will report about two patients with giant aneurysms of the basilar artery involving the origin of the PCA and a combined surgical and interventional neuroradiological approach. Preoperatively both patients showed only mild neurological symptoms (slight left hemiparesis, incomplete hemianopsia). We anastomosed the superficial temporal artery as an extracranial-intracranial bypass end-to-side to the PCA followed by clipping the PCA out of the aneurysm. Next day embolization of the aneurysm with Guglielmi ditachable coils was done. Both patients recovered without complications. An angiographic control showed no more filling of the aneurysm and a free running bypass feeding the PCA. In our opinion this combined approach is an effective method to treat giant aneurysms of the basilar artery which involve the origin of the PCA when clipping alone is impossible.
\end{abstract}

Key words: giant aneurysm, basilar artery, extracranial-intracranial bypass, posterior cerebral artery, embolization

\section{Introduction}

Giant aneurysms of the basilar artery are rare. Only $5-10 \%$ of all brain artery aneurysms are located in the vertebrobasilar circulation and only $0.5-1.5 \%$ of them are giant aneurysms with a diameter of $25 \mathrm{~mm}$ or more. They are often partially thrombosed and show atheromatous plaques. Occasionally they are broadbased with a bulbous origin encorporating the origin of the posterior cerebral artery (PCA). A rupture causing a subarachnoid hemorrhage is described in $30-60 \%$ of all giant basilar artery aneurysms. It is nearly similar compared to aneurysms of the anterior circulation. ${ }^{1,2)}$ Therefore, a repair of the aneurysm should be attempted.

Especially aneurysms of the basilar bifurcation, which incorporate the origin of PCA represent a therapeutic problem. In many of these cases neither clipping alone nor coil embolization alone will be practical without causing ischemia in the depending brain areas. We will report about two patients and a new combined surgical and interventional neuroradiological approach in the treatment of giant aneurysms of the basilar artery encorporating the origin of the PCA.

\section{Case Reports}

Case 1: A 21-year-old male came to our clinic with increasing headaches for 2 years. Furthermore, he described a transient reduction of his visual field occurring more than three times a day. The preoperative physical examination showed a slight left hemiparesis, which was not noticed by the patient. Examination of the visual field was unremarkable. Computed tomography (CT) showed a mass lesion with a diameter of $4 \mathrm{~cm}$ affecting the right midbrain and compressing the right cerebral peduncle. In the magnet- 
ic resonance (MR) imaging, this lesion could be indentified as a partially thrombosed and calcified giant aneurysm probably of the basilar artery. Cerebral angiography revealed a fusiform giant aneurysm of the basilar tip with a bulbous origin encorporating the right PCA.

Case 2: A 40-year-old female was diagnosed with an incidental aneurysm. After a ski accident with a short loss of consciousness, CT demonstrated a structure near the right midbrain which was suspected to be a giant aneurysm. The following angiography and MR imaging revealed a partially thrombosed and calcified giant aneurysm of the basilar bifurcation. The origin of the left PCA was also encorporated. First an embolization was tried. But during this procedure the PCA was partially occluded by Guglielmi detachable coils with an partial ischemia of the left occipital lobe. After this, the patient suffered from an incomplete hemianopsia and an angiographic control discovered still flow in the aneurysm. Later on our examination this patient remained with an incomplete right hemianopsia. There were no other neurological deficits.

In both cases the aneurysm was fusiform, partially thrombosed, and calcified, so that clipping of the proximal part seemed to be impossible. Therefore, we decided to choose a combined surgical and interventional neuroradiological approach.

\section{Surgery and Embolization}

All the surgical procedures were done under burst suppression by the senior author. First the superficial temporal artery (STA) was prepared for bypass. A temporal craniotomy with a subtemporal approach was used to dissect the aneurysm including the feeding vessels. After this distal part of the PCA was prepared and a clip was placed. The STA was anastomosed as an extracranial-intracranial bypass endto-side to the PCA under Doppler sonographic control. The clip was removed to the PCA at the origin from aneurysm. After the intervention both patients rested intubated, ventilated, and sedated for nearly 24 hours. Blood pressure was controlled between 100 and $120 \mathrm{mmHg}$. Three hours after the operation, CT was performed to rule out a postoperative intracerebral hemorrhage. The following days, the embolization of the aneurysm was carried out by endovascular coiling using Gugliemi detachable coils. After this procedure, an angiographic control verified complete occlusion of the aneurysm and patency of the bypass.

\section{Results}

Angiography after surgery and embolization showed a complete obliteration of the aneurysm and a free running bypass in both cases. The patients recovered without complications. CT scans 10 days after intervention proved no new abnormal findings, especially there were no new ischemic areas. Both patients could leave the hospital within 2 weeks after the operation. At discharge from hospital Case 1 showed a slight left hemiparesis, which was unchanged compared to his preoperative situation. There were no new neurological deficits. Six months later his hemiparesis improved. There was only a slight apraxia of the left hand. The patient was already working in his old profession. Case 2 suffered a postoperative new incomplete left oculomotor nerve paresis. Six months later it resolved completely. The known hemianopsia remained unchanged. This patient was working in his old profession also.

\section{Discussion}

Giant aneurysms of the basilar bifurcation are often very hard to manage. The first option of treament is the surgical clipping. Sometimes a fenestrated Drake clip must be used. Especially small saccular aneurysms with a clear neck, not thrombosed, and not encorporating branching vessels are treatable this way. ${ }^{11}$ Since the last 10 years embolization as an interventional neuroradiological endovascular approach has become more popular. This method seems to be successfull in the management of saccular aneurysms in patients who are poor grade. With this approach obliteration of the aneurysm is less frequent than with surgical clipping. In a recent study a complete obliteration of the aneurysm was achieved only in $50 \%$ of all cases. ${ }^{3,4)}$ Half the time a residual neck with the danger of rebleeding remained.

Both kinds of intervention, clipping and embolization, are often insufficient in broadbased, partially thrombosed giant aneurysms which involve the origin of the PCA. On one side the aneurysm should be completely obliterated to prevent a subarachnoid hemorrhage, on the other side the parent and branching vessels should be spared to prevent ischemia. Especially the midbrain supplying vessels should be kept patent. This problem is well illustrated in the second case described above. In this case embolization was tried first without protecting the posterior circulation. The patient sustained a partial ischemia of the medial occipital lobe causing an incomplete hemianopsia.

We demonstrated a new combined surgical and 
neuroradiological interventional approach in the treatment of broadbased giant basilar tip aneurysms. An extracranial-intracranial bypass provides sufficient blood flow into the posterior circulation. The clipping of the PCA at its origin near the aneurysm allows a complete obliteration of the aneurysm by endovascular treatment without risking an ischemia. Follow up is short (less than 1 year). It remains uncertain if coil embolization provides permanent obliteration of the aneuryms. The continual compression of the coils by the blood pulsations might lead to a partial reopening of the aneurysm so that long-term results should follow.

We conclude that our approach is a supplement to the other well-known therapy modalities, especially when neither clipping, nor embolization seems to be possible.

\section{References}

1) Drake CG: Giant intracranial aneuryms: Experience with surgical treatment in 174 patients. Clin Neurosurg 26: 12-95, 1979

2) Peerless SJ, Drake CG: Management of aneurysms of posterior circulation, in Youmans JR (ed): Neurological Surgery. Philadelphia, WB Saunders, 1986, pp 17151763

3) Raymond J: Endovascular treatment of acutely ruptured and unruptured aneurysms of the basilar bifurcation. J Neurosurg 86: 211-219, 1997

4) Vinuela F: Guglielmi detachable coil embolization of acute intracranial aneurysm: perioperative anatomical and clinical outcome in 403 patients. J Neurosurg 86: $475-482,1997$

Address reprint requests to: W. Hassler, M.D., Städtische Kliniken, $\mathrm{Zu}$ den Rehwiesen 7-9, 47055 Duisburg, Germany. 